\title{
EchoGéo
}

$47 \mid 2019$

Nouvelles géographies de la collecte

\section{La formalisation des récupérateurs à Bogota et Lima : reconnaître, réguler puis intégrer?}

\section{Mélanie Rateau et Luisa Tovar}

\section{OpenEdition}

Journals

Édition électronique

URL : https://journals.openedition.org/echogeo/16562

DOI : $10.4000 /$ echogeo. 16562

ISSN : 1963-1197

Éditeur

Pôle de recherche pour l'organisation et la diffusion de l'information géographique (CNRS UMR 8586)

Référence électronique

Mélanie Rateau et Luisa Tovar, «La formalisation des récupérateurs à Bogota et Lima : reconnaître, réguler puis intégrer? », EchoGéo [En ligne], 47 | 2019, mis en ligne le 21 avril 2019, consulté le 11 août 2021. URL : http://journals.openedition.org/echogeo/16562 ; DOI : https://doi.org/10.4000/echogeo. 16562

Ce document a été généré automatiquement le 11 août 2021.

EchoGéo est mis à disposition selon les termes de la licence Creative Commons Attribution - Pas d'Utilisation Commerciale - Pas de Modification 4.0 International (CC BY-NC-ND) 


\title{
La formalisation des récupérateurs à Bogota et Lima : reconnaître, réguler puis intégrer?
}

\author{
Mélanie Rateau et Luisa Tovar
}

\section{Introduction}

1 L'histoire de la récupération des déchets en vue de leur valorisation est liée à celle de l'urbanisation. L'Amérique latine connaît des processus rapides d'urbanisation, alimentés par une migration rurale dont les populations se caractérisent par les faibles revenus et la sous-qualification (Médina, 1999). La récupération informelle des déchets constitue alors une activité de survie dans des villes offrant peu d'emplois, marquées par la pauvreté et par le manque de programmes sociaux (id.). Les déchets sont ainsi considérés comme un gisement urbain (Cavé, 2013), dont la part valorisable représente une véritable ressource. Et ce sont les récupérateurs de déchets qui se dédient à l'activité de récupération de cette ressource, souvent en travaillant dans l'informalité. Ils opèrent sans être salariés, financés, reconnus, ni formellement chargés de ce service par les autorités concernées (Gunsilius et al. 2011 ; Gupta 2012).

2 L'informalité est présente à toutes les étapes techniques de la gestion des déchets, que sont : la collecte, le transport puis le traitement par stockage ou valorisation (Durand, 2010). Certains acteurs informels sont spécialisés dans la collecte puis l'évacuation des ordures ménagères vers les décharges sauvages, quand d'autres récupèrent les déchets organiques pour alimenter des élevages porcins. Dans cet article, nous nous intéressons aux seuls récupérateurs de déchets recyclables et non-organiques, tels que le verre, le papier, le plastique, etc... Les rapports institutionnels (GIZ, 2011 ; UN-HABITAT, 2012) plaident pour leur intégration et contre leur marginalisation sociale. Cette mise à la marge est liée, entre autre, à leur proximité aux déchets, rejets de la société de consommation et vecteurs de maladies, et à leur apparence de saleté (Médina, 1999). Cet article se concentre sur deux capitales de plus de 8 millions d'habitants, Bogota en 
Colombie et Lima au Pérou, où les récupérateurs de déchets bénéficient de politique d'intégration à la gestion formelle des déchets.

3 Le District Capital de Bogota et les cinquante municipalités de Lima, responsables des services de collecte et de transport des déchets, mettent en œuvre des processus de formalisation des récupérateurs de déchets pour que la pratique de récupération se convertisse en un service de collecte sélective. Est-ce que les processus de formalisation à l'œuvre à Bogota et à Lima permettent d'intégrer et de sortir de la marginalité les récupérateurs de déchets ? Est-ce que les récupérateurs sont bien partie intégrante de ces processus? L'hypothèse de départ est que les modèles de formalisation des récupérateurs mis en place sur les terrains étudiés découlent plus de contextes institutionnels locaux que de la prise en compte des caractéristiques des organisations de récupérateurs, ce qui est susceptible de produire de nouvelles marginalités et exclusions.

4 Cet article tente de répondre à ces questionnements à partir de données empiriques issues de cinq mois de stage en 2014, puis de cinq mois de terrain en 2015 réalisés à Lima et de trois mois à Bogota en 2016 dans le cadre du projet de recherche ORVA2D ${ }^{1}$ et à partir du travail d'accompagnement des organisations de récupérateurs réalisé par le GSEID² à Bogota depuis 2015. La méthodologie repose sur une revue de la littérature grise et scientifique, des entretiens auprès des municipalités, des ONG et des récupérateurs, ainsi que des visites et des observations de terrains. La première partie de l'article présente le tournant environnemental et social de la gestion des déchets en faveur de l'intégration des récupérateurs, puis s'attache à démontrer que le processus de formalisation est bien plus qu'une simple formalité. Il s'agit de reconnaitre, de réguler et d'intégrer les récupérateurs. La deuxième partie revient sur les processus de formalisation à l'œuvre en Colombie et au Pérou - sous la contrainte à Bogota, sous des subventions à Lima. Enfin, la dernière partie s'intéresse aux impacts sur l'informalité. Les premiers résultats révèlent la persistance d'une récupération informelle, bien que les modèles varient entre les deux terrains. 




Auteur : M. Rateau, 2017.

\section{Tournant en faveur de l'intégration des récupérateurs de déchets recyclables}

\section{Tournant environnemental et social dans la gestion des déchets}

À partir des années 1970, la gestion des déchets entame une réelle transition environnementale (Cavé, 2013) qui aboutit notamment à la nécessité de la collecte sélective. Puis, dans les années 2000, la récupération informelle des déchets recyclables fait l'objet de nombreux travaux scientifiques et institutionnels qui se positionnent en faveur de son intégration dans la gestion formelle des déchets (Cavé, 2013). Cette intégration participerait au développement durable en poursuivant des objectifs environnementaux d'augmentation des taux de recyclage et d'allongement de durée de vie des centres d'enfouissement, des objectifs économiques en alimentant l'économie circulaire et des objectifs sociaux notamment en fournissant un emploi et une nouvelle reconnaissance sociale à une population historiquement précaire et marginalisée (Médina, 1999 ; Gupta, 2012).

Navarrete-Hernández et Navarrete-Hernández (2018) ont réalisé une revue de la littérature permettant d'identifier les différentes politiques publiques à l'égard de la récupération informelle issues des cinq courants de pensées qu'ils ont différenciés. Les courants dualiste et volontariste déboucheraient sur des politiques de répression et donc de volonté d'élimination de la récupération informelle. Le courant structuraliste conduirait à un soutien faible des organisations de récupérateurs. Le courant légaliste mènerait, quant à lui, à des politiques d'ignorance. Et le courant relatif à la co- 
production aurait le potentiel de produire une politique forte d'intégration des récupérateurs. L'approche de Médina (2005) résume les politiques publiques sous quatre formes: l'ignorance, la répression, la collusion et l'intégration des récupérateurs. Aparcana (2017) se concentre sur les formes que peut prendre cette intégration. Elle peut se faire via l'implication des associations et coopératives de récupérateurs, via leur organisation en associations de base ou microentreprises ou, enfin, via le recrutement des récupérateurs informels en tant qu'agents de la gestion formelle.

7 La revue de la littérature ne met pas en évidence un modèle idéal permettant une intégration formelle des récupérateurs à la gestion officielle des déchets. De nombreux auteurs s'accordent plutôt pour affirmer que les mesures d'intégration doivent s'adapter aux contextes socio-politico-économiques propres à chaque pays.

\section{Reconnaître, réguler, intégrer}

8 La notion de formalisation employée ici est issue de l'anglais "formalization » et de l'espagnol «formalización » qui se réfèrent au passage de l'informalité à la formalité. Les organisations de récupérateurs doivent engager les démarches nécessaires à l'obtention d'un statut juridique reconnu par les autorités.

9 Ce processus, dans le cas de l'activité de récupération des déchets recyclables, est bien plus qu'une simple formalité administrative. Il s'accompagne d'une reconnaissance institutionnelle d'une activité et des personnes longtemps restées dans la marginalité (Médina, 1999). En Colombie, la Cour Constitutionnelle affirme que les « récupérateurs sont historiquement les détenteurs d'un rôle environnemental d'une très haute importance " par l'arrêt n 275 de 2011. Tandis qu'au Pérou, la Loi n 27419 précise que "l'État reconnait l'activité des récupérateurs, promeut leur formalisation et leur intégration au système de gestion des déchets... ». Ces textes dépassent le mythe du récupérateur qui ne serait qu'une "pauvre victime» (Dias et Samson, 2016). Accompagner les récupérateurs en prenant en compte leur contexte professionnel est un facteur de réussite d'un changement durable et positif (Scheinberg et al., 2006). La reconnaissance est aussi sociale. Pour ce faire, les populations sont sensibilisées à l'intérêt du tri sélectif et au travail des récupérateurs permettant d'offrir un service de collecte sélective. Le port d'un uniforme participe également à cette reconnaissance sociale. Les récupérateurs n'ont plus une apparence assimilable aux déchets (Médina, 1999), mais bien à un professionnel en exercice. 
Illustration 2 - Collecte sélective à Lima (à gauche) et à Bogota (à droite) réalisée par les récupérateurs formalisés portant leur uniforme

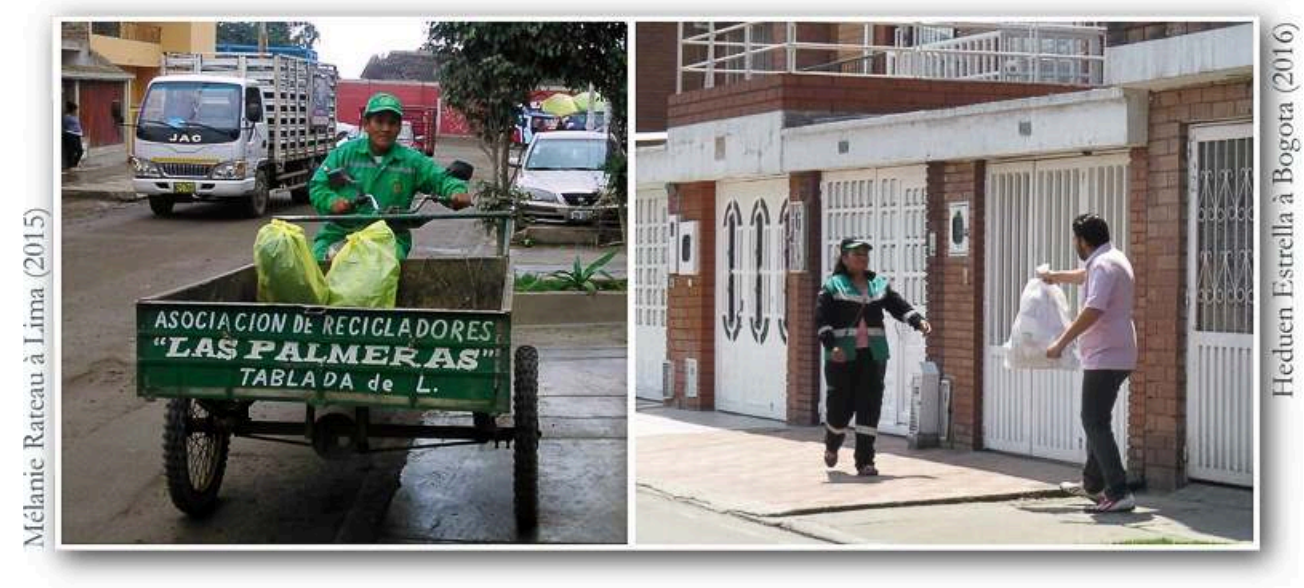

Auteurs : M. Rateau, Lima, 2015 (gauche) ; H. Estrella, Bogota, 2016 (droite)

L'activité de la récupération opérée dans l'informalité ne respecte pas toujours les règles sectorielles. Les récupérateurs informels, tant à Bogota qu'à Lima, éventrent parfois les sacs poubelles dans les rues pour en extraire les déchets recyclables, laissant le reste de leurs contenus s'épandre sur les voies. Cet exemple est une des nombreuses pratiques qui nuisent à l'environnement sanitaire urbain. Lors du processus de formalisation, les gouvernements cherchent à réguler les pratiques des récupérateurs pour ensuite les intégrer au schéma de gestion des déchets.

\section{Processus de formalisation : au-delà d'une simple formalité}

\section{En Colombie : une action en justice menée par les organisations de récupérateurs}

La reconnaissance de l'activité des récupérateurs de déchets en Colombie est le résultat d'un combat judiciaire mené par leurs organisations. L'article $n^{\circ} 25$ de la Constitution colombienne de 1991 définit que toute personne bénéficie du droit d'exercer un travail dans des conditions dignes et justes. Silvio Ruiz Grisales, leader de plusieurs organisations de récupérateurs, et l'Association des Récupérateurs de Bogotá (ARB) ont saisi la Cour constitutionnelle contre le District Capital de Bogotá et son Unité administrative spéciale des services publics (UAESP), le 23 décembre 2002, pour violation de leur droit fondamental au travail lors de l'appel d'offre pour le service de collecte et transport des déchets, qui définissait des critères qui leur était impossible de remplir.

La Cour Constitutionnelle a imposé au District Capital et à l'UAESP, au moyen de la Sentence T-724 de 2003, de garantir la participation des récupérateurs lors des futurs appels d'offre. Ce que l'UAESP n'a pas respecté. Par la résolution 114 de 2003 de l'UAESP, le règlement technique et opérationnel pour la concession du service de gestion des déchets définit que les prestataires privés de la collecte et du transport des déchets deviennent responsables de la prestation de la collecte sélective. Ce n'est qu'à 
partir de 2006 que la collecte sélective devient effective d'abord auprès de 74000 ménages, puis auprès de 700000 ménages en 2008 (Parra, 2016). Dans le même temps, seule une quarantaine de récupérateurs ont bénéficié d'un plan d'intégration au sein du centre de tri (Parra, 2016), alors que la grande majorité reste exclue du modèle de gestion des déchets. Suite à un nouveau combat judiciaire, la Cour Constitutionnelle, par l'arrêt nº 275 de 2011, a de nouveau annulé l'appel d'offre. L'UAESP se voit obligée de définir un schéma de formalisation et de régularisation de la population des récupérateurs.

Pour répondre aux arrêts de la Cour Constitutionnelle et essayer de surmonter la période de transition du régime de fourniture de service public d'assainissement dû à l'effondrement de l'appel d'offre, le District Capital a dû établir un volet zéro déchet au Plan de développement «Bogotá Humana 2012-2016». En outre, un Plan de Gestion Intégral des Déchets a été formulé en mettant l'accent sur la valorisation, visant à promouvoir : i) un changement dans les pratiques pour instaurer le tri à la source, ii) la réutilisation des déchets, iii) la régularisation et la formalisation du recyclage, la dignification du travail des récupérateurs et la promotion de leur organisation en entreprises soutenables.

\section{Un district capital bogotanais contraint à intégrer les récupérateurs}

Le plan d'intégration des récupérateurs a été rédigé pour répondre à l'arrêt n ${ }^{\circ} 275$ de 2011 de la Cour Constitutionnelle et leur garantir l'accès aux déchets recyclables. Puis, le 20 février et le 9 août 2013, s'en sont suivies les résolutions $n^{\circ} 119$ et $n^{\circ} 388$ qui adoptent la procédure de rémunération des récupérateurs de Bogotá. Suite à l'adoption de ces politiques districales, l'entreprise publique de gestion des déchets a récupéré la prestation des routes de collecte sélective qui étaient opérées par des prestataires privés, avant de les répartir entre les organisations de récupérateurs ayant le statut juridique d'organisation autorisée. Ce statut légal s'obtient suite à l'inscription individuelle de chaque récupérateur et de son association au registre public de l'UAESP, à la signature d'un accord de coresponsabilité et à l'enregistrement de l'association à la Superintendance des Services Publics Ménagers.

, cette politique locale devient nationale par l'approbation du décret $n^{\circ} 1077$ qui reconnait la valorisation des déchets comme une activité complémentaire du service publique de gestion des déchets. Il est suivi en 2016, par le décret n596 qui approuve le régime transitoire pour la formalisation des récupérateurs. Ces derniers ont un délai de 5 ans pour se conformer aux exigences techniques, administratives, commerciales et financières qui leur incombent pour répondre aux appels d'offre de prestation d'un service public. Les organisations de récupérateurs doivent assumer la gestion de la comptabilité, le contrôle des routes avec des systèmes de géoréférencement, la gestion des plaintes et des réclamations, entre autres exigences pour s'aligner sur les entreprises privées de prestation de service public.

De ce processus de formalisation, la rémunération pour le service rendu représente l'une des avancées les plus significatives pour la reconnaissance du travail des récupérateurs (Parra, 2015). Ce que les intéressés confirment ${ }^{3}$. Ils sont rémunérés en fonction de la quantité de matières recyclables collectées suite à leur passage dans un des centres de pesage autorisés. Le montant de leur rémunération est fixé en fonction des coûts évités par la non-prise en charge des déchets recyclables par le service de 
collecte-enfouissement. Puis les récupérateurs tirent un second revenu de la vente de ces déchets. Toutefois, l'accès à cette ressource urbaine pour les récupérateurs semble compromis. Ils redoutent la future mise en concurrence avec les prestataires privés : «Déjà trois ans de formalisation sur les cinq. Et dans deux ans, ils vont tous nous détruire $»^{4}$.

\section{Au Pérou : une légifération aidée par la mobilisation d'une ONG}

Depuis les années 1990, le recyclage des déchets au Pérou est encadré par une législation dense, mais trop stricte envers l'activité de récupération des déchets (Durand, 2010). D’après la Loi Générale des Déchets de 2000, seules les entreprises enregistrées sous le statut d'Entreprise Prestataire de Services des Déchets Solides ou bien d'Entreprise Commercialisant des Déchets Solides, ont le droit de trier, de vendre et de commercialiser les déchets en vue de leur recyclage. Or, les nombreuses conditions à remplir pour l'obtention de ces statuts sont inaccessibles aux récupérateurs, les empêchant ainsi d'exercer leur activité dans le respect de la législation. En conséquence, les municipalités ont mené des actions de répression à leur encontre, notamment en leur confisquant leur tricycle et leur charrette.

Dans ce contexte, l'ONG Ciudad Saludable est née en 2002 avec l'objectif de promouvoir la reconnaissance de l'activité des récupérateurs de déchets recyclables. Pour ce faire, elle a soutenu la création de la Fédération Nationale des Récupérateurs du Pérou (FENAREP) en 2005 avec qui elle a pu entamer des discussions auprès du Ministère de l'environnement (Samson, 2009). Suite à des désaccords entre la FENAREP et l'ONG, notamment au sujet du zonage de la collecte sélective ${ }^{5}$, une nouvelle organisation de récupérateurs dénommée le Mouvement national des récupérateurs du Pérou (MNRP) est créée en 2008. Cette même année, une commission composée du Ministère de l'environnement, de l'ONG Ciudad Saludable, du MNRP et des représentants de la société civile, est chargée de la rédaction du projet de loi (Samson, 2009) visant à l'encadrement de la pratique de la récupération des déchets.

19 La Loi n²9419, dite Loi du Recycleur, est adoptée le 7 octobre 2009. Elle vise à inciter la formalisation des récupérateurs et la mise en place de la collecte sélective grâce à leur intégration à la gestion municipale.

\section{Des subventions ministérielles pour inciter les municipalités liméniennes}

20 La nouvelle législation régulant l'activité des récupérateurs de déchets recyclables désigne les municipalités districales comme responsables de la formalisation de ces acteurs et de la mise en œuvre de programme de tri et de collecte sélective des déchets recyclables (PSF-RS). Pour les inciter à s'engager dans cette démarche, le gouvernement a lancé une campagne de subvention récompensant l'atteinte d'objectifs chiffrés fixés annuellement. Par exemple, en juillet 2014, si $25 \%$ des ménages urbains du district étaient inscrits au PSF-RS, la municipalité percevait une subvention ministérielle (República del Perú 2014).

21 La formalisation et le PSF-RS sont adaptables à chaque municipalité car définis et adoptés par ordonnances municipales. Seules les grandes étapes du processus administratif sont encadrées par la Loi. Ce processus débute par une campagne de 
recensement des récupérateurs. Ces derniers s'enregistrent et reçoivent un justificatif nécessaire pour accéder à une formation obligatoire, à une campagne de vaccination et à une affiliation gratuite à une assurance santé. La formalisation se concrétise lors de leur inscription au registre municipal en mentionnant obligatoirement de quelle association ils dépendent. Les récupérateurs ne peuvent se formaliser en tant que récupérateur indépendant. Ils doivent s'organiser en association pour effectuer la collecte sélective au sein du PSF-RS.

Les municipalités doivent simultanément mettre en œuvre leur PSF-RS pour assigner des zones de collecte aux récupérateurs formalisés. Elles cartographient ces zones, y assurent la sensibilisation des ménages au tri sélectif et relèvent les coordonnées de ceux désirant participer au programme. Ensuite, chaque récupérateur assure la collecte sélective dans la zone que lui attribue la municipalité. Cette collecte s'effectue en porteà-porte en recevant des mains des ménages inscrits au PSF-RS, leurs sacs de tri. La nouvelle régularité des revenus ainsi que l'acceptation sociale sont les principaux attraits de la formalisation pour les récupérateurs, comme une récupératrice l'explique: "Je me suis formalisée parce que la municipalité nous offrait une opportunité pour que les voisins nous reconnaissent comme les personnes que nous sommes, des travailleurs et pour qu'on n'ait plus cette peur que n'importe quel jour ils aillent faire une battue et nous confisquent le tricycle, tout ça ${ }^{6}$.

\section{Des modèles aux impacts contrastés sur l'informalité}

\section{Persistance de l'informalité}

Les résultats des processus de formalisation à Bogota et à Lima révèlent la persistance d'une récupération informelle. Prenons l'exemple de Lima. L'impact de la nouvelle règlementation y est minime, tant en nombre de récupérateurs intégrés qu'en tonnage de collecte sélective. À Comas, se sont 40 récupérateurs qui sont formalisés ou en cours de formalisation, soit seulement $2 \%$ des récupérateurs d'après les estimations de l'ONG Ciudad Saludable (Ruiz Ríos, 2010). Ces récupérateurs collectent près de 300 tonnes de déchets recyclables par an, soit moins de $1 \%$ des déchets recyclables produits par le district. Les récupérateurs informels sont bien plus nombreux que les formels et les tonnages totaux qu'ils captent sont probablement plus importants. 
Illustration 3 - Récupérateurs informels à Lima (à gauche) et à Bogota (à droite) ouvrant les sacs poubelles à la recherche de déchets recyclables

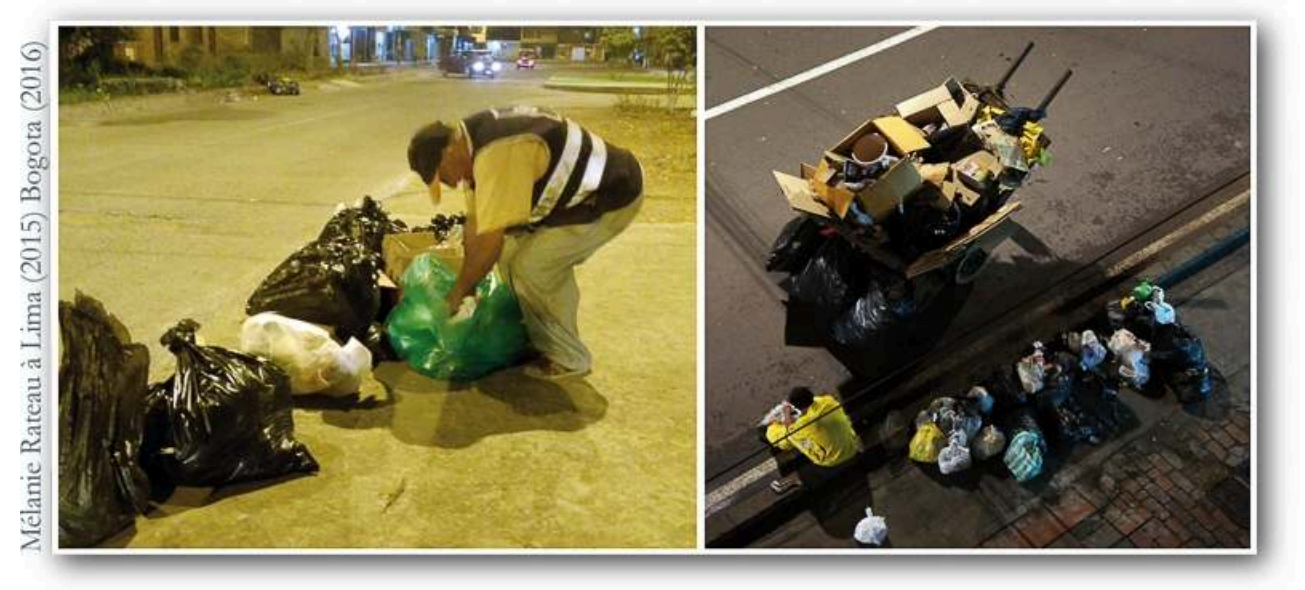

Auteurs : M. Rateau, Lima, 2015 (gauche). M. Rateau, Bogota, 2016 (droite) publiques à l'égard de la récupération informelle. Les modèles de Bogota et de Lima sont deux modèles distincts d'intégration qui ne rentrent pourtant pas parfaitement dans les cases proposées par Navarrete-Hernández et Navarrete-Hernández (2018). Ce qui tient notamment des contextes préexistants à la politique de formalisation et d'intégration. À Bogota, la collecte sélective était opérée par des prestataires privés avant que la nouvelle règlementation impose un régime transitoire d'intégration des récupérateurs. À Lima, la majorité des districts ne pratiquait pas la collecte sélective, mais seulement une collecte des ordures en mélange. À Bogota, les récupérateurs ont un délai de cinq ans pour se mettre à niveau des prestataires privés afin de pouvoir rentrer dans un schéma de mise en concurrence. À Lima, c'est la réglementation qui a été simplifiée pour être à la portée des récupérateurs.

Exiger une mise à niveau des récupérateurs entraine des effets pervers. Les grandes organisations se consolident alors que les plus petites craignent de devenir des coquilles vides au fur et à mesure que leurs membres rejoignent les plus grandes ${ }^{8}$. Le risque à terme est que de nombreuses organisations n'arrivent pas à suivre ce 
développement forcé et retombent dans l'informalité. Le choix de simplifier la réglementation, tel qu'opéré à Lima, offre l'avantage de conserver des caractéristiques de flexibilité et d'autonomie propres à l'informalité, que les récupérateurs ne souhaitent pas abandonner (ESCAP, 2015). Toutefois, le manque d'assistances techniques, financières et organisationnelles risque de limiter le développement économique des organisations des récupérateurs en les enfermant dans la seule activité de récupération. Ce rôle d'assistance est parfois mené par les ONG lorsque les municipalités les engagent pour les accompagner dans le processus de formalisation. Par exemple, l'ONG Alternativa dans son projet Recicla para la vida (2012-2015) offrait des micro-crédits et un accompagnement à la création d'entreprise de prétraitement et de commercialisation des déchets aux organisations de récupérateurs souhaitant développer leurs activités.

\section{Conclusion}

Les politiques d'intégration des récupérateurs de déchets à Bogota et à Lima sont issues des contextes socio-politico-économiques locaux. À Bogota, la collecte sélective préexistait au combat judiciaire des organisations des récupérateurs. Leur intégration se fait par leur mise à niveau sur les prestataires privés lors d'un régime transitoire de cinq ans, dans le but d'une mise en concurrence lors des futurs appels d'offre pour la prestation du service de collecte sélective. À Lima, peu de municipalités mettaient en place des mesures de tri sélectif avant la Loi du Recycleur. L'intégration des récupérateurs s'avère une opportunité pour la fourniture de ce nouveau service. La règlementation a été simplifiée pour faciliter leur formalisation.

Les processus de formalisation à l'œuvre permettent de reconnaitre une activité et des travailleurs, de réguler les pratiques et de les intégrer à la gestion formelle des déchets. Mettre fin à l'informalité de ce secteur n'est pas à la portée de ces processus qui ne concernent que les récupérateurs de déchets recyclables et non-organiques. Ils participent plutôt à mettre fin à la marginalisation de la pratique de récupération de ces déchets. À Bogota, cette pratique était déjà institutionnalisée mais à Lima, les municipalités sensibilisent activement les populations. La collecte sélective s'y fait en récupérant les sacs de tri des mains des ménages. La fouille dans les sacs poubelles accessibles sur la voirie devrait, quant à elle, être amenée à diminuer et à se marginaliser.

La reconnaissance des récupérateurs comme des professionnels du secteur de la gestion des déchets serait un facteur de réussite d'un changement durable et positif (Schneinberg et al., 2006). Pourtant, cette reconnaissance n'est que la première étape d'un régime transitoire à Bogota. Les organisations de récupérateurs doivent rentrer dans une logique de développement forcé pour les convertir en organisation respectant les mêmes exigences que celles demandées aux prestataires privés de service public. Les récupérateurs avancent dans le processus de formalisation en craignant de retomber dans l'informalité lors de la dernière étape théorique de mise en concurrence. À Lima, la simplification de la réglementation et le manque d'accompagnement des récupérateurs risquent de les enfermer dans la seule activité de récupération, sans leur offrir l'opportunité de se développer. La Loi régulant leur activité est issue, en grande partie, de la mobilisation d'une ONG. Ce qui explique peut-être un modèle de formalisation dépendant des ONG pour l'assistance aux organisations. 
31 sont qu'à leurs débuts. Actuellement, à Bogota, les organisations n'en sont qu'aux premières étapes du processus. À Lima, la nouvelle formalité a du mal à attirer les récupérateurs. Et l'engagement des municipalités dans ces démarches dépend des subventions ministérielles. Il est nécessaire de suivre l'évolution de ces politiques et processus dans la durée pour en connaitre les conséquences sur la marginalisation et l'intégration des récupérateurs.

\section{BIBLIOGRAPHIE}

Aparcana S., 2017. Approaches to formalization of the informal waste sector into municipal solid waste management systems in low- and middle-income countries: Review of barriers and success factors. Waste Management, 1 mars 2017, vol. 61, pp. 593-607.

Cavé J., 2013. La gestion disputée d'un mal public impur :économie politique des ordures. Thèse de doctorat en architecture, aménagement de l'espace, Université Paris-Est, Champs-sur-Marne, $459 \mathrm{p}$.

Dias S. M. et Samson M., 2016. Informal economy monitoring study sector report : waste pickers. WIEGO, Cities inclusive, IEMS, $60 \mathrm{p}$.

Durand M., 2010. Gestion des déchets et inégalités environnementales et écologiques à Lima : entre durabilité et vulnérabilité. Thèse de doctorat de Géographie et Aménagement de l'espace, Université de Rennes 2.

Escap, 2015. Valuing waste, transforming cities, Economic and social commission for Asia and the Pacific. United Nations publication, $129 \mathrm{p}$.

GIZ, 2011. Recovering resources, creating opportunities: integrating the informal sector into solid waste management. $54 \mathrm{p}$.

Gunsilius E., Chaturvedi B., Scheinberg A., Coad A. et Garcia Cortes S., 2011. The economics of the informal sector in solid waste management. Eschborn, CWG, GIZ, $36 \mathrm{p}$.

Gupta S. K., 2012. Intégrer le secteur informel pour une meilleure gestion des déchets. Secteur Privé \& Développement, 2012, n5, (coll. «Les déchets: quels enjeux pour les pays en développement?»), pp. 12-17.

Médina M., 1999. Reciclaje de desechos solidos en America Latina. Frontera norte, vol. 11, n²1, pp. 7-31.

Medina M., 2005. Serving the unserved: informal refuse collection in Mexico. Waste Management \& Research, 2005, vol. 23, n5, pp. 390-397.

Navarrete-Hernandez P. et Navarrete-Hernandez N., 2018. Unleashing waste-pickers' potential : supporting recycling cooperatives in Santiago de Chile. World Development, vol. 101, pp. 293-310.

Parra F., 2015. Reciclaje: jsí, pero con recicladores! Gestión pública del aprovechamiento con inclusión de recicladores: un nuevo paradigma en el manejo de los residuos en Bogotá. Nota Técnica de WIEGO 9 (Políticas Urbanas).

EchoGéo, 47 | 2019 
Parra F., 2016. De la dominación a la inclusion : la población recicladora organizada como sujeto politico. Tesis de doctorado en estudios politicos y relaciones internacionales, Universidad Nacional de Colombia, Bogota, $624 \mathrm{p}$.

Republica del Peru, 2014. Decreto Supremo No033-2015-EF. Aprueban los procedimientos para el cumplimiento de metas y la asignación de los recursos del plan de incentivos a la mejora de la gestión y modernización municipal del año 2015.

Ruiz Ríos A., 2010. Por la ruta del reciclaje en el Perú. Estudio socioeconómico de la cadena de reciclaje. Lima, Ciudad Saludable.

Samson M., 2009. Rechazando a ser excluidos : la organizacion de los recicladores en el mundo. WIEGO, Cambridge, $116 \mathrm{p}$.

Scheinberg, A., Anschütz, J. et Van de Klundert A., 2006. Waste pickers: poor victims or waste management professionals?. Paper n56, CWG-WASH Workshop. Kolkata, India, 1-5 February 2006.

Sspd, 2018. Système Unique d'information disponible en ligne. http://www.sui.gov.co (page consultée en octobre 2018)

Uaesp, 2012. Plan de Inclusión de la Población y Organizaciones de Recicladores en la Gestión Pública de Residuos Sólidos Reciclables en Bogotá D.C. (Secretaria de Hábitat, Ed.). Bogotá D.C.

UN-HABITAT, 2012. Recycling and disposal of municipal solid waste in low and middle-income countries: Perspectives for municipal managers and environment agencies. Nairobi: UN-HABITAT, 224 p.

\section{NOTES}

1. Le projet de recherche ORVA2D (ORganisation de la VAlorisation des Déchets dans les pays en Développement) était piloté par le laboratoire ESO (UMR 6590) de l'Université du Mans sur financement de l'AFD.

2. GSEID, Groupe de recherche en socio-économie, institutions et développement de l'Université Nationale de Colombie.

3. Entretien collectif avec les récupérateurs d'une organisation du quartier Kennedy à Bogota, réalisé par Mélanie Rateau et Heduen Estrella, le 29/01/2016.

4. Propos d'un récupérateur formalisé lors d'une audience publique, recueillis par Luisa Tovar, le 27/07/2018.

5. Entretien avec Juan Herrera, président de la FENAREP à Lima, réalisé par Mélanie Rateau le $16 / 04 / 2014$.

6. Entretien avec une récupératrice de la municipalité de San Martin de Porres, dans le nord de Lima, réalisé par Mélanie Rateau le 19/03/2014.

7. Entretien avec une récupératrice de la municipalité de San Martin de Porres, dans le nord de Lima, réalisé par Mélanie Rateau le 25/05/2014.

8. Entretien avec des récupérateurs de Bogota réalisé par Heduen Estrella, le 10/03/2016. 


\section{RÉSUMÉS}

Les modèles de gestion des déchets en Colombie et au Pérou connaissent un tournant en faveur de l'intégration des récupérateurs informels des déchets. Le District Capital de Bogota et les cinquante municipalités de Lima mettent en place des processus de formalisation qui sont bien plus qu'une simple formalité. Il s'agit de reconnaitre, de réguler et d'intégrer les récupérateurs de déchets. L'étude des processus à l'œuvre dans ces deux villes révèle des modèles de formalisation distincts aux impacts contrastés sur l'informalité et la marginalité d'une pratique et de la population qui s'y dédie.

Waste management models in Colombia and Peru are at a turning point in favor of the integration of informal waste pickers. The Capital District of Bogota and Lima's fifty municipalities are putting in place formalization processes that are more than just a formality. It involves recognizing, regulating and integrating wastepickers. The study of the processes at work in these two cities reveals distinct models of formalization with contrasting impacts on the informality and the marginality of a practice and the population dedicated to it.

\section{INDEX}

Mots-clés : formalisation, récupérateur informel, gestion des déchets, Bogota, Lima

Keywords : formalization, informal wastepicker, waste management, Bogota, Lima

Thèmes : Sur le Champ - Sur le Terrain

\section{AUTEURS}

\section{MÉLANIE RATEAU}

Mélanie Rateau, melanie.rateau@hotmail.com, est doctorante en géographie au Latts (UMR 8134), ENPC.

\section{LUISA TOVAR}

Luisa Tovar, lftovarc@unal.edu.co, est doctorante en sciences économiques à l'Université Nationale de Colombie. 